\title{
TEST SUITE MINIMIZATION FOR EMBEDDED NONDETERMINISTIC FINITE STATE MACHINES
}

\author{
Nina Yevtushenko \\ Tomsk State University \\ 36 Lenin av., 634050 Tomsk, Russia \\ yevtushenko@elefot.tsu.ru
}

\section{Ana Cavalli}

National Institute of Telecommunications

9, rue Charles Fourier, 91011, Evry Cedex, France

Ana.Cavalli@int-evry.fr

\author{
Ricardo Anido* \\ University of Campinas \\ Cx Postal 6167 UNICAMP, 13083-970 Campinas SP, Brazil \\ ranido@dcc.unicamp.br
}

\begin{abstract}
This paper presents a method for minimizing test suites for embedded, nondeterministic Finite State Machines. The method preserves the fault coverage of the original test suite, and can be used in conjunction with any technique for generating test suites. The minimization is achieved by detecting and deleting redundant test cases in the test suite. The proposed method is an extension of the work presented in [Yevtushenko et al., 1998].
\end{abstract}

Keywords: Embedded testing, Automatic test generation, finite state machines, non-determinism.

${ }^{*}$ Work supported by FAPESP and Pronex/Finep/SAI N: 76.97.1022.00 


\section{INTRODUCTION}

One important aspect of automatic test generation that has been investigated recently is the derivation of tests for a component embedded within a complex system. This problem is known as gray-box testing, embedded testing ([ISO, 1991]) or testing in context ([Petrenko et al., 1996b]), and corresponds to a situation quite common in practice, where the system to be tested (the component) is part of a larger system, and can only be tested in the context of another part assumed to be fault-free (the context). A number of approaches have been developed for testing in context ([Petrenko et al., 1996b, Petrenko et al., 1996a, Lee et al., 1996, Lima and Cavalli, 1997, Lima and Cavalli, 1998, Petrenko et al., 1997, Yevtushenko et al., 1998]), some of which are heuristic and do not guarantee complete fault coverage ([Lee et al., 1996]). Other approaches deliver complete test suites with respect to various fault domains (i.e., w.r.t. sets of possible implementations of a component under test). Examples of the latter approach are methods which start with a test suite either derived by classical procedures such as W-, Wp, UIO, SC methods ([Vasilevsky, 1973, Chow, 1978, Fujiwara et al., 1991, Vuong et al., 1989, Yannakakis and Lee, 1995, Lee and Yannakakis, 1996]) or given by human experts; the derived test suite is afterwards reduced without loss of its completeness.

In this paper we propose a method for minimizing a test suite for communicating nondeterministic Finite State Machines (FSMs) while maintaining its fault-coverage. We consider a specification system composed of two communicating FSMs. One of these machines, called component, is the machine that needs testing. The other machine, called context, describes the behavior of the part of the system which is assumed to be correctly implemented. Component, context or the overall system may be nondeterministic.

The method proposed extends the work presented in ([Yevtushenko et al., 1998]), for deterministic FSMs, to nondeterministic FSMs. For each test sequence of a given test suite, we determine a regular set of internal traces that can be induced within an arbitrary implementation system. The regular sets thus obtained completely characterize the fault detection power of the test suite. We then present a procedure for determining a minimal proper subset of the test suite which accepts all nondeterministic behaviors accepted by the original test suite while maintaining its fault-detection power. The rest of the paper is structured as follows. Section 2. presents some basic notions. Section 3. analyzes the conditions under which a test case can be removed from a test suite without loss of its completeness. Section 4. describes how the problem of minimizing a given test suite can be reduced to determining a minimal column coverage of a Boolean matrix, and in Section 5. we present some conclusions. 


\section{PRELIMINARIES}

This section presents some basic notions on composition of FSMs and conformance testing.

\subsection{FINITE STATE MACHINES}

A Finite State Machine (FSM, often simply called a machine throughout this paper) is an initialized (possibly, nondeterministic) machine denoted by a 5-tuple $A=\left(S, X, Y, h, s_{0}\right)$, where $S$ is a finite nonempty set of states with $s_{0} \in S$ as the initial state, $X$ is a finite nonempty set of inputs, $Y$ is a finite nonempty set of outputs, and $h$ is a behavior function, $h: S \times X \rightarrow P(S \times Y)$ where $P(S \times Y)$ is a set of all nonempty subsets of $S \times Y$. FSM $A$ is deterministic if $|h(s, x)|=1$ for all $(s, x) \in S \times X$. The machine is observable if for all $(s, x) \in S \times X$ and $y \in Y$ it holds that $|(s, y)|(s, y) \in h(s, x) \mid \leq 1$. In the usual way, the function $h$ is extended to a function on the set $S \times X^{*}$ with results in the set $P(S \times Y *)$ where $X^{*}$ is the set of all finite sequences in $X$ containing the empty sequence $\epsilon$. The function $h$ has two projections $h^{s}$ and $h^{y}$, usually called the next state function and the output function. FSM $A$ is said to be connected if each state of $A$ is reachable from the initial state, i.e. for each state $s$ of $A$ there exists an input sequence $\alpha$ such that $s \in h^{s}\left(s_{0}, \alpha\right)$.

Given sequences $\alpha=x_{1} \ldots x_{k} \in X^{*}$ and $\beta=y_{1} \ldots y_{k} \in Y^{*}$, the sequence $x_{1} y_{1} \ldots x_{k} y_{k}$ is called a trace at state $s \in S$ of FSM $A=(S, X, Y, h, s 0)$ if $\beta \in h^{2}(s, \alpha)$. We call the sequence $x_{1} y_{1} \ldots x_{k} y_{k}$ a trace of $A$ if $\beta \in$ $h^{2}(s, \alpha)$. Given two states $s$ of FSM $A=\left(S, X, Y, h, s_{0}\right)$ and $t$ of FSM $B=\left(T, X, Y, g, t_{0}\right)$, state $s$ is said to be equivalent to state $t$, written $s \leq t$, if $h^{2}(s, \alpha)=g^{2}(t, \alpha)$ for each input sequence $\alpha \in X^{*}$; otherwise, state $s$ is distinguishable from state $t$ and the sequence $\alpha$ is said to distinguish state $s$ from state $t$. Machines $A$ and $B$ are equivalent if their initial states are equivalent, written $A \equiv B$; otherwise, they are distinguishable, written $A \neq B$. An input sequence is said to distinguish two machines if it distinguishes their initial states. Machine $A$ is said to be reduced if every pair of its states is distinguishable. It is well known that each machine $A$ is equivalent to some reduced observable FSM that is called a reduced observable form of machine $A$ ([Starke, 1972, Luo et al., 1994]). In other words, given FSMs $A$ and $B$ over the same input alphabet, $A$ and $B$ are equivalent if they exhibit the same behavior under all input sequences; otherwise, they are distinguishable. Protocol conformance testing is often formalized as a problem of testing whether an implementation FSM is equivalent to a given reference FSM. 


\subsection{COMPOSITION OF FSM'S}

We consider a system composed by two FSMs, as shown in Figure 1. We will use the expression system under test to designate a system composed by some implementation Imp of the component Comp and the context $C$. For the sake of simplicity, we consider pairwise disjoint sets $X, Y, U$ and $V$. The system under test has always a single message in transit, i.e. the environment submits the next input only when the system has produced an output to the previous input. We refer to symbols of the alphabet $X$ as external inputs, to symbols of the alphabet $Y$ as external outputs, and to symbols of the alphabets $U$ and $V$ as internal actions. As it is shown in a number of publications ([Yevtushenko et al., 1998]) such a composition is general enough to discuss problems of testing in context. When there are no livelocks in the composition, we can derive the composite FSM of FSMs $C$ and Comp using various algorithms ( [Petrenko et al., 1996b, Lima and Cavalli, 1997]).

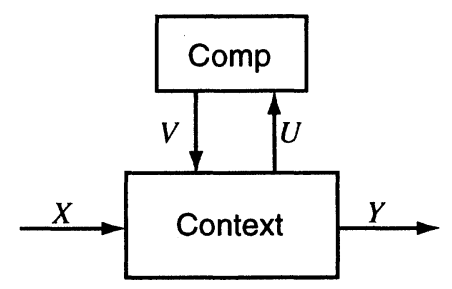

Figure 1 System model

Example. We use as an example of a coffee shop system which is inspired from the one proposed in [Petrenko et al., 1998]. The coffee shop is composed by a waiter and a coffee machine. We have modified the global behavior of the coffee shop to introduce nondeterminism. The nondeterminism is caused by the context, represented in our example by a forgetful waiter, which may forget a request for coffee, as described below. In order to simplify the description of the components we consider that internal actions are denoted by elements of disjoint sets $U$ and $V$. The behavior of the coffee shop is as follows: if a customer introduces money $(M)$, the shop answers with thanks $(T)$ and is ready to receive the following inputs: $M$, which it refuses saying No $(N)$; Espresso Please $\left(E_{p}\right)$, producing two nondeterministic outputs: either Yes $(Y)$, remaining in the same state (forgets the request), or Espresso Served $\left(E_{s}\right)$, returning to the initial state. At the initial state a customer can also request a coffee $\left(E_{p}\right)$, but the answer in this case is sorry (S). The coffee-machine has two inputs and two outputs. When the waiter presses Button (B) at the initial state 0 the coffee-machine responses turning on a Lamp ( $L)$. If Button $(B)$ is pressed at the state 1 the coffee-machine produces Espresso (E). An input Coin (C) causes a looping transition and an output Espresso $(E)$ at each state. 
The FSMs representing the waiter and coffee-machine are presented in Figure 2 .

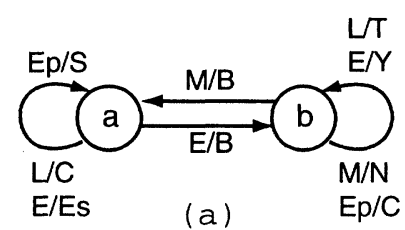

Figure 2 (a) Context FSM Waiter and (b) Component FSM CoffeeMachine

The combined system of waiter and coffee machine has no livelocks and its composite FSM RS is shown in Figure 3.

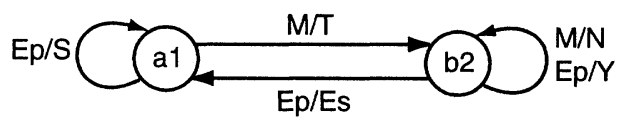

Figure 3 The FSM RS modeling the behavior of the coffee shop

\subsection{FAULT MODEL FOR TESTING IN CONTEXT}

We consider a reference system that is a composition of two FSMs, and assume that at most one of the two machines, named the component machine, denoted Comp, can be faulty. The other machine, named the context, denoted $C$, is fault-free. In other words, any implementation system is a composition of the context and some (possibly faulty) implementation component machine Imp combined, as shown in Figure 1. We also assume the behavior of the reference system as well as the behavior of any (possibly faulty) implementation system is described by a reduced observable FSM with at most $n$ states. The set of all possible implementation systems is called a fault domain, and is denoted $\Re_{n, C}$.

To test a system with a nondeterministic behavior each test sequence usually is submitted to the system under test several times, under diverse conditions, until a test engineer verifies the system has produced each possible output to the test sequence. Under this so-called "all-weather conditions" assumption one can use the same notion of a complete test suite for deterministic and nondeterministic machines.

A test suite for the reference FSM $R S$ is a finite set of finite input sequences of $R S$. A test suite for the FSM $R S$ is complete w.r.t. the fault domain $\Re_{n, C}$ if for any FSM $B \in \Re_{n, C}$ such that $B$ and the reference machine are distinguishable the test suite has a sequence distinguishing the machines; otherwise, the test suite is not complete w.r.t. the fault domain $\Re_{n, C}$. 
One can use a straightforward approach to derive a complete test suite w.r.t. the fault domain $\Re_{n, C}$ : explicitly enumerate all FSMs of $\Re_{n, C}$ and for each FSM $B \in \Re_{n, C}$ that is distinguishable from the reference machine determine a sequence distinguishing the machines. The set of all distinguishing sequences is a complete test suite w.r.t. $\Re_{n, C}$. When it is impossible to explicitly enumerate all FSMs of the set $\Re_{n, C}$ we can expand $\Re_{n, C}$ to the fault domain $\Re_{n}$ comprising any FSM that has a reduced observable form with $n$ states, and use existing methods for complete test derivation w.r.t. the fault domain $\Re_{n}$. Below we sketch a method proposed in [Luo et al., 1994]; in fact, this method is similar to the well known W or Wp-method for deterministic FSMs when the reference FSM is reduced and completely specified.

Let $R S$ be a reduced connected FSM with $n$ states. A finite subset $V$, $|V| \leq n$, including the empty sequence, is called a state cover of $R S$ if for each state $s \in S$ there exists an input sequence $\alpha$ such that $s \in h^{s}(s, \alpha)$. We further denote $V X$ the set obtained by concatenating each sequence of the set $V$ with each sequence of the set $X$. The set $V X$ is usually called a transition cover of the FSM $R S$ since each transition of the FSM $R S$ is traversed with an appropriate sequence of the set $V X$. Here we notice that differently from deterministic FSMs the state cover $V$ of a nondeterministic FSM $R S$ can have less sequences than number $n$ of states of $R S$ ([Luo et al., 1994]).

Given a state $s \in S$ of the FSM $R S=\left(S, X, Y, h, s_{0}\right)$, a finite set $W_{s}$ of finite input sequences is said to be a state identifier of $s$ if for each state $p \in S$, $p \neq s$, there exists an input sequence $\alpha$ distinguishing state $s$ from state $p$, i.e. $h^{y}(s, \alpha) \neq h^{y}(p, \alpha)$. An input sequence is said to be a distinguishing sequence for the FSM $R S$ if it is a state identifier for every state of $R S$. Similar to the W-method when a state identifier is fixed for each state of the FSM $R S$, a procedure for derivation of a complete test suite w.r.t. the fault domain $\Re_{n}$ comprises two phases.

In the first phase, part $T_{1}$ of a test suite is derived. $T_{1}$ verifies that an implementation FSM has at least $\mathrm{n}$ states and that each state identifier correctly identifies the corresponding state. This part of the test suite is obtained by concatenating each sequence $\alpha$ of the state cover set $V$ with each sequence of the state identifier for each last state of $\alpha$, that is for each state where $\alpha$ takes the reference FSM, starting from the initial state. If the implementation FSM has the reference output response to each input sequence of the set $T_{1}$ then every state of the reference FSM has a corresponding state in the implementation FSM with the same state identifier.

Part $T_{2}$ of the test suite derived in the second phase. $T_{2}$ checks whether each transition in the implementation FSM is correctly implemented. The set $T_{2}$ is obtained by concatenating each sequence $\alpha \in V X$ with each sequence of the state identifier for each state where $\alpha$ takes the reference FSM from the initial state. Merging $T_{1}$ and $T_{2}$ we obtain a complete test suite $T S=T_{1} \cup T_{2}$ 
w.r.t. the fault domain $\Re_{n}$. Each sequence that is a prefix of another sequence can be deleted from $T S$, without loss of completeness of $T S$ w.r.t. the fault domain $\Re_{n}$. If we then apply each sequence of $T S$ to an implementation under test, several times, to observe each possible response, and if the set of responses coincides with that of the reference machine then we conclude the implementation has a reference behavior.

Example. The reference machine in Figure 3 is nondeterministic, reduced and observable. We assume the waiter is fault-free and any system with a (possibly faulty) coffee-machine has an observable reduced form with at most two states. An input sequence $M$ is a distinguishing sequence of the reference FSM. We select a state cover set $V=\{\epsilon, \mathrm{M}\}$. Then $T_{1}=\left\{\mathrm{E}_{\mathrm{p}}, \mathrm{M}, \mathrm{ME} \mathrm{E}_{\mathrm{p}}, \mathrm{MM}\right\}$ while $T_{2}=\left\{\mathrm{E}_{\mathrm{p}} \mathrm{M}, \mathrm{MM}, \mathrm{ME} \mathrm{E}_{\mathrm{p}} \mathrm{M}, \mathrm{MMM}\right\}$, and $T S=\left\{\mathrm{E}_{\mathrm{p}} \mathrm{M}, \mathrm{ME} \mathrm{E}_{\mathrm{p}} \mathrm{M}, \mathrm{MMM}\right\}$.

A test suite $T S$ complete w.r.t. the fault domain $\Re_{n}$ is also complete w.r.t. the fault domain $\Re_{n, C}$. However, it usually is redundant, because some of the test cases included are (unnecessarily) testing only the context, which is assumed fault-free. Furthermore, some test cases may be testing parts of the implementation already tested by other test cases.

Given a test suite $T S$, complete w.r.t. the fault domain $\Re_{n}$, a proper subset $T$ of $T S$ is complete w.r.t. $\Re_{n, C}$ if, for every possible implementation FSM $B \in \Re_{n, C}$, when $B$ has the expected set of output responses to every sequence of $T$, it has also the expected set of output responses to every sequence of $T S$. As shown in [Yevtushenko et al., 1998], the detecting power of each test case can be characterized through a set of internal traces that are detectable by the test case. We can delete from $T S$ every test case traversing only transitions of the context, since the set of internal traces that are detectable by this test case is empty, i.e. the test case checks nothing in the component. For example, the suffix MM of the test case MMM tests nothing in the component ([Lima and Cavalli, 1997, Yevtushenko et al., 1998]). Therefore, the test suite $\left\{E_{p} M, M E_{p} M, M\right\}$ is also complete w.r.t. the fault domain $\Re_{n, C}$. If the test case $\alpha \in T S$ traverses transitions in which the component is involved a more detailed analysis of the test case must be performed. In the next sections we show that $T S \backslash\{\alpha\}$ is complete w.r.t. $\Re_{n, C}$ if the following two properties hold: (i) there exists a test case $\alpha \in T S \backslash\{\alpha\}$ that induces an unexpected external input for each internal trace that can induce an unexpected external output to $\alpha$; (ii) for each reference output sequence $\beta$ to $\alpha$ there exists a test case $\alpha \in T S \backslash\{\alpha\}$ which induces an internal trace such that the system under test produces the output response $\beta$ when $\alpha$ is submitted.

\section{MAINTAINING THE FAULT COVERAGE}

In this section, we analyze the conditions to reduce the set $T S$ by deleting some of its sequences without loss of its completeness w.r.t. the fault domain 
$\Re_{n, C}$. Given an external input sequence $\alpha$, an internal trace $\gamma=x_{1} y_{1} \ldots x_{k} y_{k}$ over alphabets $U$ and $V$ is said to be detectable by $\alpha$ if, when $\alpha$ is applied to a system under test where the component $\operatorname{Imp}$ has the trace $x_{1} y_{1} \ldots x_{k} y_{k}$, the system produces an unexpected output response to $\alpha Y C L 98$. Otherwise, a trace is said to be undetectable by $\alpha$. Given a set $T$ of external input sequences, an internal trace $\gamma$ over alphabets $U$ and $V$ is said to be detectable by $T$ if there exists $\alpha \in T$ such that the internal trace $\gamma$ is detectable by $\alpha$.

In [Yevtushenko et al., 1998] we show that any prolongation of a trace detectable by $\alpha$ is also detectable by $\alpha$, and that the set of all internal traces detectable by $\alpha$, in the case of systems composed by deterministic context and component FSMs, is a regular set ([Hopkroft and Ulman, 1979]). The same results are valid in the case of nondeterministic context and/or component.

\subsection{DETERMINING DETECTABLE INTERNAL TRACES}

Procedure 1 below describes a step by step derivation of the regular set of detectable internal traces for given context. The key construction of the procedure is the acceptor $L C(\alpha)$ of all possible traces that can be induced by an external input sequence when the context is combined with any implementation of the component machine Comp. Traces detectable by $\alpha$ are designated in the $(U \cup V)$-projection of the acceptor $L C(\alpha)$ by a dead state fail. Below we briefly sketch how the acceptor $L C(\alpha)$ can be constructed.

Given an external input sequence $\alpha=x_{1} \ldots x_{k}$, the acceptor $L C(\alpha)$ can be derived step by step by use of the deterministic Label Transition System (LTS) $L C$ representing all traces of the context $C$. States of the acceptor $L C\left(x_{1} \ldots x_{k}\right)$ are states of $L C$. Given an input $x_{1}$, we construct the acceptor $L C\left(x_{1}\right)$ starting from the initial state of the $L C$. There is a transition labeled with $x_{1}$ from the initial state to state $p$ if $x_{1}$ takes LC from the initial state to state $p$. For two intermediate states $p$ and $r$, there is a transition labeled with internal action $a \in U \cup V$ if there is a transition labeled with a from state $p$ to state $r$ in $L C$. There is a transition labeled with $y \in Y$ in the $L C\left(x_{1}\right)$ from intermediate state $p$ to a final state if there exists an outgoing transition labeled $y$ from state $p$. The acceptor $L C\left(x_{2}\right)$ is constructed at each final state of the acceptor $L C\left(x_{1}\right)$ and so on. The final states of the acceptor $L C\left(x_{k}\right)$ are declared the final states of the acceptor $L C\left(x_{\alpha}\right)$.

\section{Procedure 1 Derivation of a regular set of detectable internal traces.}

Input: The composite FSM $R S$ of a reference system, the deterministic LTS $L C$ representing all traces of the context $C$ and an external input sequence $\alpha$. Output: The regular set $D(\alpha)$ of internal traces detectable by $\alpha$.

Step 1. Construct the acceptor $L C(\alpha)$ using the deterministic context LTS $L C$.

Step 2. For each path of the acceptor $L C(\alpha)$ from the initial state to a final state such that the projection of the sequence labeled the path is not a trace of the reference FSM, replace the final state with a final state fail. 
Step 3. If for some transition labeled with an external input $x \in X$ or with an internal action $v \in V$, all the subsequent paths have a final state fail then replace the final state of the transition with final state fail.

Step 4. Construct the $(U \cup V)$-projection of the obtained acceptor by a subset construction, replacing with a designated fail-state without outgoing transitions each subset that has the fail-state. Construct the regular set $D(\alpha)$ as the set of all sequences of the $(U \cup V)$-projection labeled paths from the initial to the fail state.

By construction of the set $D(\alpha)$, the following statements hold.

Proposition 1 Given a reference system $R S$ and an implementation system IS with a component Imp, the system IS produces an unexpected response to the input sequence $\alpha$ if and only if the set of traces of the machine Imp intersects the set $D(\alpha)$ derived by Procedure 1 ([Yevtushenko et al., 1998]).

Corollary 2 Given a reference FSM RS, an implementation system IS, a complete test suite $T S$ w.r.t. the fault domain $\Re_{n, C}$ and a sequence $\alpha \in T S$, let the system IS have an unexpected response to the sequence $\alpha$. If for each trace $u_{1} v_{1} \ldots u_{k} v_{k} \in D(\alpha)$ there exists a sequence $\gamma \in T S \backslash\{\alpha\}$ such that the set $D(\alpha)$ comprises a prefix of the trace $u_{1} v_{1} \ldots u_{k} v_{k}$, then the system IS has an unexpected response to some sequence of the set $T S \backslash\{\alpha\}$.

In fact, let all the conditions of Corollary 2 hold. If the $I S$ has an unexpected response to the sequence $\alpha$ then the set of traces of the component Imp has a trace $u_{1} v_{1} \ldots u_{k} v_{k}$ of the set $D(\alpha)$ (Proposition 1), along with its prefix $u_{1} v_{1} \ldots u_{l} v_{l}, l \leq k$, being in the set $D(\gamma)$ for some sequence $\gamma \in T S \backslash\{\alpha\}$ i.e. $I S$ has an unexpected response to the sequence $\gamma$.

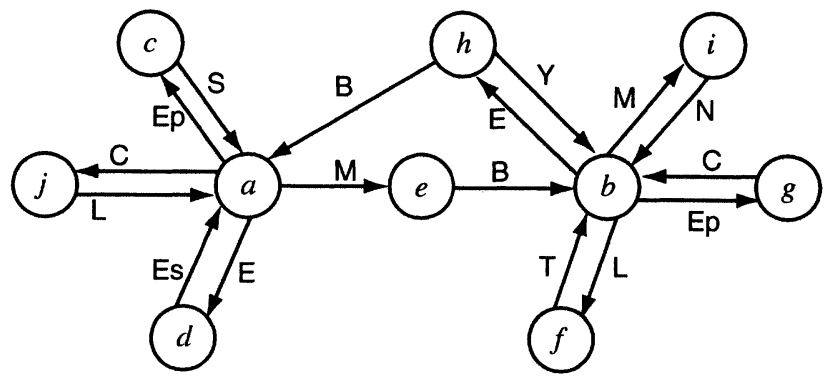

Figure 4 The context LTS LC

Example. Consider an input sequence $M \in T S$. The deterministic context LTS $L C$ is shown in Figure 4. The acceptor $L C(\mathrm{M})$ and its $(U \cup V)$-projection with the designated fail-state are shown in Figures 5 and 6 . If the component implementation $I m p$ has a trace BE then the implementation system $I S$ has an unexpected output response $Y$ to input $M$. On the other hand, if the component 
implementation Imp has no trace BE then the implementation system IS always produces the expected output response $T$ to the input $M$. Thus, the implementation system $I S$ always produces the expected output response T to the input $\mathrm{M}$ if and only if the component implementation Imp answers with output $E$ to the input $B$. Thus, $D(M)=\{B E\}$. Similarly, we obtain $D\left(\mathrm{E}_{\mathrm{p}} \mathrm{M}\right)=\{\mathrm{BE}\}$ and $D\left(\mathrm{ME}_{\mathrm{p}} \mathrm{M}\right)=\left\{\mathrm{BE}, \mathrm{BLCL}, \mathrm{BLCEB}(\mathrm{LC})^{*} \mathrm{EBE}\right\}$. By direct inspection, one can assure that if the implementation system $I S$ has only expected output responses to the input sequence $M E_{p} M$ then the implementation component machine $I m p$ has no traces of the set $D\left(\mathrm{ME}_{\mathrm{p}} \mathrm{M}\right)$, i.e. $I S$ has the expected output responses to the input sequences $E_{p} M$ and $M$.

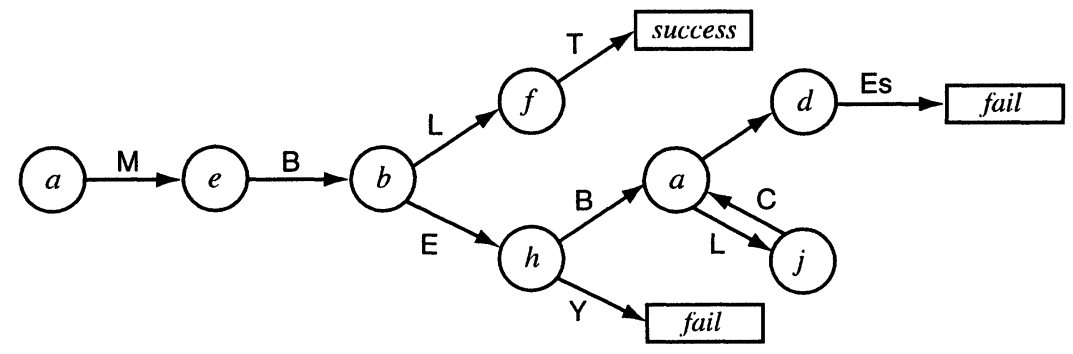

Figure 5 Acceptor LC(M)

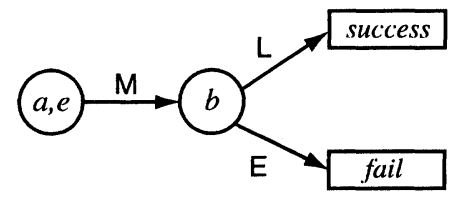

Figure $6(U \cup V)$-projection of acceptor LC(M)

Corollary 2 specifies a necessary and sufficient condition for minimizing a test suite while maintaining its fault detection power when the context and the component are deterministic. Although this condition is insufficient in the case of nondeterministic systems, since the minimized test must also accept all possible behaviors accepted by the original test suite. That property is analyzed in the next section.

\subsection{PRESERVING ALL POSSIBLE BEHAVIORS}

Given an external input sequence $\alpha=x_{1} \ldots x_{k}$, let $L C(\alpha)$ be an acceptor of all internal traces that can be induced in the context when $\alpha$ is submitted to an implementation system. Let also $\beta=y_{1} \ldots y_{k}$ be an output sequence that can be produced by the reference system in response to $\alpha$. We denote $C(\alpha, \beta)$ the regular set of $(U \cup V)$-projections of all sequences that label paths 
from the initial to the final state in $L C(\alpha)$ and have the $(X \cup Y)$-projection $x_{1} y_{1} \ldots x_{k} y_{k}$. If a system under test has produced an output response $\beta$ to the sequence $\alpha$, it means that the set of traces of the implementation component machine intersects the set $C(\alpha, \beta)$.

Procedure 2 Derivation of the regular set of all internal traces which are possible in an implementation system with a given reference trace.

Input: The composite FSM $R S$ of a reference system, the LTS $L C$ representing all traces of the context $C$, an external input sequence $\alpha$ and the output response $\beta$ of the reference system $R S$ to $\alpha$.

Output: The regular set $C($ alpha, $\beta)$ of all internal traces which are possible to occur in an implementation system with the reference trace $(\alpha, \beta)$.

Step 1. Construct the acceptor $L C(\alpha)$ using the deterministic context LTS $L C$. Step 2. For each path of the acceptor $L C(\alpha)$ from the initial state to a final state such that the projection of the sequence labeled the path is a trace $(\alpha, \beta)$, replace the final state with a designated state success.

Step 3. Construct the $(U \cup V)$-projection of the obtained acceptor by a subset construction replacing with a designated state success each subset that has the state success. Derive the regular set $C(\alpha, \beta)$ as the set of all sequences of the $(U \cup V)$-projection labeled paths from the initial to the state success.

Proposition 3 Given two external input sequences $\alpha$ and $\beta$, the sets of output responses $h^{y}\left(s_{0}, \alpha\right)$ and $h^{y}\left(s_{0}, \delta\right)$ of the reference system $R S$ to the sequences $\alpha$ and $\delta$, let IS be an implementation system such that the set of output responses of IS to $\delta$ coincides with $h^{y}\left(s_{0}, \delta\right)$. If for every $\beta \in h^{y}\left(s_{0}, \alpha\right)$ there exists a sequence $h^{y}\left(s_{0}, \delta\right)$ such that each sequence in the set $C(\delta, \lambda)$ has a prefix in the set $C(\alpha, \beta)$ then the set of output responses of IS to $\alpha$ coincides with $h^{y}\left(s_{0}, \alpha\right)$.

As a corollary to Proposition 3, another condition for a proper subset $T$ of the complete test suite $T S$ to provide a complete set of reference outputs to each test case in $T S$ can be established.

Corollary 4 Given the reference FSM RS, an implementation system IS, a complete test suite $T S$ w.r.t. the fault domain $\Re_{n, C}$ and a sequence $\alpha \in T S$, let the system IS have only expected output responses to the sequence $\alpha$. Let also for each reference output response $\beta$ to the sequence $\alpha$, there exist a sequence $\delta \in T S \backslash\{\alpha\}$ and $\lambda \in h^{y}\left(s_{0}, \delta\right)$ such that each trace in the set $C(\delta, \gamma)$ has a prefix in the set $C(\alpha, \beta)$. If for each sequence $\delta \in T S \backslash\{\alpha\}$, the set of output responses of IS to $\delta$ coincides with that of the reference system then the set of output responses of the system IS to the sequence $\alpha$ also coincides with that of the reference system.

Example. The set $C\left(\mathrm{ME}_{\mathrm{p}} \mathrm{M}, \mathrm{TYN}\right)=\{\mathrm{BLCE}\}$ while $C\left(\mathrm{ME}_{\mathrm{p}} \mathrm{M}, \mathrm{TE}_{\mathrm{s}} \mathrm{T}\right)=$ $\left\{\mathrm{BLCEB}(\mathrm{LC})^{*} \mathrm{EBL}\right\}$. In other words, an implementation system $I S$ has all expected output responses to the input sequence $M E_{p} M$ if and only if the implementation component machine has the trace $B L C E$ as well as at least one trace of the regular set $B L C E B(L C){ }^{*} E B L$. 


\section{MINIMIZATION OF A TEST SUITE}

By combining the results of Corollaries 2 and 4 we obtain a sufficient condition for a proper subset of a complete test suite to be also complete w.r.t. the fault domain $\Re_{n, C}$.

Proposition 5 Given a reference FSM RS, a complete test suite TS w.r.t. the fault domain $\Re_{n, C}$, let $\alpha \in T S$. The set $T S \backslash\{\alpha\}$ is also complete w.r.t. $\Re_{n, C}$ if the following conditions hold.

a) For every trace $u_{1} v_{1} \ldots u_{k} v_{k} \in D(\alpha)$, there exists $\delta \in T S \backslash\{\alpha\}$ such that the set $D(\delta)$ comprises a prefix of the trace $u_{1} v_{1} \ldots u_{k} v_{k}$.

b) For every reference output response $\beta \in h^{y}\left(s_{0}, \alpha\right)$ there exist $\delta \in$ $T S \backslash\{\alpha\}$ and $\lambda \in h^{y}\left(s_{0}, \delta\right)$ such that for every trace $u_{1} v_{1} \ldots u_{k} v_{k} \in$ $C(\delta, \lambda)$, the set $C(\alpha, \beta)$ comprises a prefix of the trace $u_{1} v_{1} \ldots u_{k} v_{k}$.

Example. In our working example, only a single input sequence $M E_{p} M$ of the test suite induces two reference output responses. Thus, the set $\left\{M E_{p} M\right\}$ is a test suite complete w.r.t. the fault domain $\Re_{n, C}$.

Proposition 5 shows that a given test suite can be minimized by examining the regular sets detectable by the test cases and the sets of internal traces providing reference output sequences. The problem of comparing arbitrary regular expressions is out of the scope of this paper. When the regular sets $C$ and $D$ derived by Procedures 1 and 2 are finite; in that case, similarly to [Yevtushenko et al., 1998], we can reduce the problem of test minimization to the problem of determining a minimal column coverage in a Boolean matrix, as described below.

Given a complete test suite $T S$, let $D(T S)$ be the set of internal traces detectable by the set $T S$ derived by Procedure 1. For each $\alpha \in T S$ that induces at least two reference output responses and each $\beta \in h^{y}\left(s_{0}, \alpha\right)$, we derive the set $C(\alpha, \beta)$ of internal traces which provide the external trace $(\alpha, \beta)$ (Procedure 2) and denote $C(T S)$ the collection of such sets. We construct a Boolean matrix $B$ as follows. Rows of the matrix $B$ correspond to sequences of the prefixes of all sequences in the test suite $T S$; columns of $B$ correspond to the items in $D(T S)$ and in $C(T S)$. That is, the columns of $B$ are all internal traces detectable by $T S$, and all subsets $C(\alpha, \beta)$ of internal traces induced by each trace $(\alpha, \beta)$ of the reference FSM $R S$ for each $\alpha \in T S$ providing at least two reference output sequences.

Element $b$ corresponding to a test sequence $\alpha \in T S$ and an internal trace $\gamma \in D(T S)$ has value ' 1 ' if and only if there exists a prefix of $\gamma$ detectable by $\alpha$. Element $b$ corresponding to a test sequence $\alpha \in T S$ and a subset $C(\delta, \lambda), \delta \in T S$, has value ' 1 ' if and only if there exists a response $\beta$ of the reference FSM RS to $\alpha$ such that each sequence in the set $C(\alpha, \beta)$ has a prefix 
in the set $C(\delta, \lambda)$. The set of rows of the matrix corresponding to its minimal column coverage is a test suite complete w.r.t. the fault domain $\Re_{n, C}$.

\section{CONCLUSION}

In this paper, the approach proposed in [Yevtushenko et al., 1998] is extended to a system of communicating nondeterministic FSMs. The system under test is composed of two FSMs, a context that is assumed to be fault-free and a component that needs testing. We assume that the behavior of both the reference and the implementation system are described by observable FSMs. In order to minimize a given test suite, complete under the above assumptions, we construct two regular sets. One of them is the set of all internal traces detectable by each test case, i.e. a set of internal traces which cause an unexpected output response of an implementation system. Another regular set is the set of all internal traces that cause a given reference output to the test case. If a system under test produces every reference output response to a test case one guarantees that the component machine has at least one trace of such set for each reference output response. After the regular sets have been derived the problem of determining a minimal subset of a given test suite that is also complete w.r.t. the chosen fault domain can be solved as a problem of finding a minimal column coverage of a Boolean matrix. We can apply the approach for test minimization w.r.t. the reduction relation. In this case, similar to a system of communicating deterministic finite state machines the Procedure 2 becomes unnecessary. The proposed method can also be used to reduce a test suite given by a human expert while preserving its fault detection power under an assumption that the context is fault-free.

\section{References}

[Chow, 1978] Chow, T. S. (1978). Test software design modeled by finite state machines. IEEE Transactions on Software Engineering, 4(3):178-187.

[Fujiwara et al., 1991] Fujiwara, S., v. Bochmann, G., Khendek, F., Amalou, M., and Ghendamsi, A. (1991). Test selection based on finite state models. IEEE Transactions on Software Engineering, 17(6):591-603.

[Hopkroft and Ulman, 1979] Hopkroft, J. E. and Ulman, J. D. (1979). Introduction to automata theory, languages and computation. Addison-Welsey, NY.

[ISO, 1991] ISO (1991). Information technology, Open systems interaction, Conformance testing methodology and framework. International Standard IS-9646.

[Lee et al., 1996] Lee, D., Sabnani, K. K., Kristol, D. M., and Paul, S. (1996). Conformance testing of protocols specified as communicating finite state 
machines - a guided random walk based approach. IEEE Transations on Communications, 44(5):631-640.

[Lee and Yannakakis, 1996] Lee, D. and Yannakakis, M. (1996). Principles and methods of testing finite state machines, a survey. IEEE Transactions, 84(8):1090-1123.

[Lima and Cavalli, 1997] Lima, L. P. and Cavalli, A. R. (1997). A pragmatic approach to generating test sequences for embedded systems. In 10th IWTCS, pages 125-140.

[Lima and Cavalli, 1998] Lima, L. P. and Cavalli, A. R. (1998). Application of embedded testing methods to service validation. In submitted to 2 nd IEEE Intern. Conf. On Formal Engineering methods.

[Luo et al., 1994] Luo, G., Petrenko, A., , and v. Bochmann, G. (1994). Selecting test sequences for partially-specified nondeterministic finite state machines. In 7th IWTCS, pages 95-110.

[Petrenko et al., 1997] Petrenko, A., , and Yevtushenko, N. (1997). Testing faults in embedded components. In 10th IWTCS, pages 125-140.

[Petrenko et al., 1998] Petrenko, A., , and Yevtushenko, N. (1998). Solving asynchronous equations. In Joint International Conference FORTE/PSTV98, pages 231-247.

[Petrenko et al., 1996a] Petrenko, A., Yevtushenko, N., , and v. Bochmann, G. (1996a). Fault models for testing in context. In 1st Joint International Conference FORTE/PSTV96, pages 125-140.

[Petrenko et al., 1996b] Petrenko, A., Yevtushenko, N., v. Bochmann, G., and Dssouli, R. (1996b). Testing in context: framework and test derivation. Computer communications, 19:1236-1249.

[Starke, 1972] Starke, P. H. (1972). Abstract automata. American Elsevier Publishing Company, Inc. New York.

[Vasilevsky, 1973] Vasilevsky, M. P. (1973). Failure diagnosis of automata. Cybernetics, (4):653-665.

[Vuong et al., 1989] Vuong, S. T., Chan, W. W. L., and Ito, M. R. (1989). The uio-method for protocol test sequence generation. In IFIP TC6 Second International Workshop on Protocol Test Systems, pages 161-175.

[Yannakakis and Lee, 1995] Yannakakis, M. and Lee, D. (1995). Testing finite state machines: fault detection. Journal of Computer and System Sciences, (50):209-227.

[Yevtushenko et al., 1998] Yevtushenko, N., Cavalli, A. R., and Lima, L. P. (1998). Test minimization for testing in context. In 11th IWTCS, pages 127-145. 